\title{
COVID-19 - Modeling and Parameter Estimation for Brazil and Portugal: How Predictable is the Future?
}

\author{
J. M. R. SOUZA ${ }^{1 *}$, T. Y. MIYAOKA ${ }^{2}$, C. F. D. KUNZ ${ }^{3}$ and J. F. C. A. MEYER ${ }^{4}$
}

Received on August 2, 2020 / Accepted on April 22, 2021

\begin{abstract}
This work addresses the COVID-19 pandemic on two fronts: proposing a system of ordinary differential equations to model it and fitting this model to Brazilian and Portuguese data. It presents estimations to important parameters for the infection dynamics, such as the percentage of asymptomatic individuals, and it stresses out that non-biological human aspects, for example, cultural, social, and economic, are not only impacted by the pandemic but also impact the pandemic dynamics itself. We state that, despite significant variations in the parameters, due to those human elements present in the contemporary pandemic, and despite the strong nonlinearities of the problem, wise human intervention is possible and able to minimize human losses. We show that the mortality rate does not behave as one would expect for a biological problem, independent of cultural aspects, and we also point to possible dates for the peaks of infection in both countries depending on the control of the transmissibility.
\end{abstract}

Keywords: pandemic in human societies, parameters estimation, modelling constrained by uncertainties.

\section{INTRODUCTION}

Never in the contemporary history of humankind have the limits of knowledge and social and economic mobilization been so defied as in the past 12 months. Although we have seen pandemics in the past, we completely forgot them. The time between the events was large enough to erase them from our collective memories.

However, never before have there been so many people that know enough mathematics with which they could model, simulate, and analyse pandemic scenarios. But are we, the people that

\footnotetext{
*Corresponding author: Juliana Marta Rodrigues de Souza - E-mail: jmrsa1@iscte-iul.pt

${ }^{1}$ ISCTE - Instituto Universitário de Lisboa, Departamento de Matemática, Av. das Forças Armadas, 1649-026, Lisboa, Portugal - E-mail: jmrsa1 @iscte-iul.pt https://orcid.org/0000-0003-4062-3635

${ }^{2}$ SEMANTIX, Av. Eusébio Matoso, 1375, 14th floor, 05424-160, São Paulo, SP, Brasil - E-mail: tiagoyuzo@gmail.com https://orcid.org/0000-0003-2735-3044

${ }^{3}$ FIAS - Frankfurt Institute for Advanced Studies, Ruth-Moufang-Strasse, 1, 60438, Frankfurt, Main, Germany - E-mail: kunz@ fias.uni-frankfurt.de https://orcid.org/0000-0002-7193-9002

${ }^{4}$ Departamento de Matemática Aplicada, IMECC, Universidade Estadual de Campinas, R. Sérgio Buarque de Holanda, 651, 13083-859, Campinas, SP, Brasil - E-mail: joni@ime.unicamp.br https://orcid.org/0000-0001-8002-7075
} 
know enough mathematics, fully aware of the major distinctions between mathematically modeling a pandemic in humans versus mathematically modeling a pandemic in any other species? Humans societies are able, to a vast extent, to change their habits. And different societies facing the same matter will change or restrain their social patterns in distinct ways. In ways not only related to their level of concern for the matter in question but also concerned with how their change itself impacts their social and economic lives. In modeling a pandemic in humans, to what degree can we disregard culture, and to what degree are we able to consider it?

Considering these particularities, we are going to propose a model for Coronavirus dynamics in humans via a nonlinear system of Ordinary Differential Equations (ODE), analyse its parameters and their meanings, fit it to Brazilian and Portuguese data, study the parameters obtained using Nonlinear Least Squares Minimization, and present some of the possible future scenarios based on what probably happened so far.

\section{THE MODEL}

We aimed at drawing the simplest System of Ordinary Differential Equations model that could capture the most striking shades of COVID-19 dissemination among humans according to the vast literature recently produced.

On the one hand, the outlined populational compartments had to be enough in number and sufficiently characterized to depict subtleties inherent to the disease dynamic's aspects that seemed to propel its spread. On the other hand, it was necessary to keep the number of parameters as little as possible because only a small share of them have values characteristic of the virus in the human population despite social and cultural differences and safety measures adopted. We will think through the SARS-COV-2 parameters in detail in Section 3. In this section, we are going to focus on the modeling aspects considered. The well-known SIR model was taken as a starting point for the population compartments, for more details see [6]. $S$ denotes Susceptible, I, Infected and $R$, Removed. From now on, when we refer to the original SIR model, we will mention its compartments as $\bar{S}, \bar{I}$ and $\bar{R}$, respectively, to distinguish these original classes from the ones we are going to use. Its basic lines depict the simplest infection dynamic while maintaining the possibility of one being removed from the Susceptible set, $\bar{S}$, and from the Infected set, $\bar{I}$, even if only temporarily.

We decided to divide the original Removed, $\bar{R}$, compartment between Recovered, $R$, and deceased, $D$. Although $D$ could initially be thought of as a fixed percentage of infected individuals only, it is one population compartment for which we actually have data. And data show that the lethality, $\mu$, usually considered as a constant, in the case of the duo human-Corona virus, is actually not a fixed percentage. In some scenarios, as we show in Section $5, \mu$ even decreased as the number of infections rises.

Furthermore, splitting $\bar{R}$ in $R$ and $D$ allows us to account for an eventual loss of immunity at a rate $\delta$, new waves of infection, and an endemic scenario caused by it. It is probable that the time span needed to see the impact of the loss of immunity parameter would also require a logistic 
term to account for population growth dynamics. We disregard population growth dynamics because it would increase the number of nonlinear terms in the model, therefore numerical effort in simulations. And also because we are focusing on the first year of infection and counting on a vaccine.

The last, and we believe the most important, modification we inserted in the SIR model was splitting the Infected population, $\bar{I}$. Every infection will be first accounted for in the Latent population, $L$, infected but not infectious. And $L$ comprises two fates: becoming Asymptomatic, $A$, meaning being infectious but not being at any risk due to the infection, or becoming what one could regard as a classical Infected person, $I$, infectious, with symptoms and facing the risk of serious health complications.

Let $\beta$ be the rate for one becoming infectious, either $A$ or $I$. The parameters more intrinsically related to human biological structure and its response to Covid-19 are the rates for loss of immunity, becoming infectious, and recuperation. These are the more transversely consistent parameters independent of the different cultural groups we focus on. Therefore, for simplicity, $\beta$ is taken as a constant in both of our applications of the model.

The percentage of $L$ that will become symptomatic, $\sigma_{I}$, and therefore the percentage of $L$ that will become asymptomatic, $\left(1-\sigma_{I}\right)$, is crucial to the pandemic's development. We will further explain the two major aspects on which we base the last assertion and how they are related. The first aspect is that Asymptomatic people can easily ignore the state they are in and therefore behave more carelessly. Although their lack of symptoms could make them a smaller source of contagion, this behavior would favor the infection spreading. In this sense, we are working with a basic parameter of infection $\alpha$, for which we get a rate $\alpha S$ of infections once the Susceptibles have contact with symptomatic people, and a rate $v \alpha S$ of infections once the Susceptibles have contact with asymptomatic people. Approaching the matter in this way, and for reasons that will be discussed in the next section, we will reduce the number of parameters we need to estimate in order to fit the model to the available data sets. Secondly, asymptomatic people will be usually not accounted for in data, particularly in countries where health resources, tests, and hospital capacity, are overwhelmed by symptomatic people. And the missing data can mislead us to a flattened data curve, depending on the $\sigma_{I}$ value.

The last two parameters, $\gamma_{A}$ and $\gamma_{I}$, are the rates of recuperation for asymptomatic people and symptomatic people, respectively. We have already mentioned that these two parameters are more strongly correlated to biological factors and therefore should be more independent from social, cultural and economic aspects. We are quite sure of this assumption for the rate of recovery for asymptomatic people. Once they have no symptoms, the average number of days they need to recover from the infection should be reasonably robust. This is not the case for symptomatic people, a percentage of whom might not even recover due to a lack of access to the health care system, an important factor to consider when calculating the lethality of Covid-19. This will be further discussed in the next section. 
Thus concludes the presentation and justification of our model characteristics that led to six Ordinary Differential Equations modelling the time variation of six population compartments, SLAIRD, see System 2.1.

$$
\begin{array}{r}
\frac{d S}{d t}=-\alpha(v A+I) S+\delta R \\
\frac{d L}{d t}=\alpha(v A+I) S-\beta L \\
\frac{d A}{d t}=\left(1-\sigma_{I}\right) \beta L-\gamma_{A} A \\
\frac{d I}{d t}=\sigma_{I} \beta L-\left(\gamma_{I}+\mu\right) I \\
\frac{d R}{d t}=\gamma_{A} A+\gamma_{I} I-\delta R \\
\frac{d D}{d t}=\mu I
\end{array}
$$

If $P_{\text {total }}$ is the total population of the analysed country, System 2.1 with initial condition $\left(S_{0}, L_{0}, A_{0}, I_{0}, R_{0}, D_{0}\right)=\left(P_{\text {total }}-1,0,0,1,0,0\right)$ leads us to a, Initial Value Problem. Although the given $\left(S_{0}, L_{0}, A_{0}, I_{0}, R_{0}, D_{0}\right)$ agrees with the data, it is worth mentioning that it is not necessarily true. Countries receiving initial cases from abroad could have $I_{0}>1$ even if the cases are not registered on the same day.

\section{PARAMETERS}

Social, cultural, economic, and possibly geographical aspects, such as weather conditions, may impact the model parameters. For that reason, we state that only a small share of parameters are characteristic of the virus in the human population. It has to be clear that modelling the spread of a disease in the human population is, in a sense, completely different from modelling any other population. If there is a dangerous infection process occurring, humans to a certain extent may modify their habits to avoid what could be thought of as the natural outcome of a pandemic. The last sentence is corroborated by the fact we are modeling the pandemic to understand it, make predictions about it, and propose safety measures to minimize its effects. Furthermore, distinct cultures may respond differently to the same pandemics because of social features such as the acceptable level of physical proximity and resilience for handling social distancing measures.

Firstly, since the Coronavirus depends on human proximity to infect new people, it relies on human contact and movement to spread. And human contact and movement may happen for many reasons, from displays of affection to taking a crowded bus. This is a good aspect if we believe human societies can cope with restrictions to get later gratification, minimize human deaths. And it is also a very complex matter because people have to understand the necessity of socializing as minimum as possible, although that might mean missing important human connections. In Brazil, the awareness process has an extra challenge because it requires a duel, not only with people not wanting to believe their life is endangered but also with the government that encour- 
ages them not to believe it, endangering them even more. The hypotheses that are assumed to get an optimized economic balance influence the pandemic outcome.

Although one may not expect a mathematical model for a pandemic process to include such political consideration, a political bias is certainly included when models are used to show people how their behaviour can influence the spread of the virus and, in turn, their lives. Also, a possible good side-effect of it might be an increase in the perception of one's autonomy. Even if convincing people of staying at home because they understand it is the best thing to do, is not sufficient to create conditions for them to do it, at least it might encourage them to question their government's decisions.

Although our system doesn't have a specific equation for people practicing social distancing, this type of damage reduction, by reducing people's movement, can be modeled by it. The six population compartments in the model refer to a singular status concerning the infection. Either a person is susceptible, latent, asymptomatic, symptomatic, recovered, or deceased. That means that if we had a sufficiently large stock of tests for a whole population in a given point of time, their results would fall into one of the compartments. Now, if we introduce an equation for those practicing social distancing, we would be creating a category that could include individuals that make up the other five compartments, namely $S, L, A, I$, and $R$. Therefore we would have more nonlinearities. And more parameters of contagion, plus the rates of entrance into, and exits from the compartments, would have to be estimated. But, if the data itself does not depict the infection scenario perfectly concerning the population status about the infection, and we still have to keep in mind the sub notification [7], one can quickly conclude the level of difficulties that an equation for social distancing population would bring. Luckily, there is another way to prove the impact that the sanitary measures have on the spread of the virus.

In this paper, the parameter $\alpha$ will be responsible for enabling us to understand the impact of social distancing and isolation measures on the spread of COVID-19. Once we understood that, although we did not have a specific population with restricted interactions, the restricting interactions were our main way of intervening in the infection evolution, and therefore flatten the curve, we became aware that by exclusively studying the impact of this parameter variation we could obtain a good fit for data available and thus make predictions. After several essays, we decided to work with $\alpha$ 's whose value is different in distinct periods of time. The extreme points of these intervals of time correspond to points of inflection in the curve of the daily number of cases from the data set. The number of plateaus in this piecewise constant function of time, $\alpha(t)$, would be doubled if we considered that asymptomatic people would infect susceptible people in a fashion completely independent from $\alpha$. It, therefore, makes sense that different traits of behaviour and infectivity, combined, would lead to the difference in the contagion from symptomatic and from asymptomatic people. Considering that $\alpha(t)$ expresses the mean contagion in a homogeneous population, and that this parameter embodies sanitary behaviour measures, we understood it was sufficient to work with a scale parameter, $v$, to obtain the information we needed about contagion from asymptomatic without doubling the number of parameters that compose $\alpha(t)$. 
The next question is: $v>1, v=1$ or $v<1$ ? In order to answer this question, we used numerical techniques described in the next section (see Subsection 4.1). The answer and its impacts in the results will be presented in Section 5. The same procedure was applied to the estimation of $\sigma_{I}$, with one main difference; for $\sigma_{I}$, a very good initial guess was available [2].

The average rate at which a latent person becomes infectious, $\beta$, was taken to be the same for asymptomatic and symptomatic people. As we said, although prior health, and therefore historical and social, conditions may impact this value for a specific individual, we have reasons to believe that this is one of the more stable parameters across different populations because it is highly biological. To intervene in it be challenging, if possible at all.

A very similar analysis to the one we just stated for $\beta$ can be done for $\gamma_{A}, \gamma_{I}$ and $\delta$. As we already mentioned, $\gamma_{I}$ is the parameter that impacts peoples' lives the most, and the one that is most dependent on social and economic factors such as access to Intensive Care Unity treatment. Consequently, in a nonlinear way, $\gamma_{I}$ and $\mu$ are related. When thinking of $\gamma_{A}$, it is worth pointing out that the shorter time one is sick, even if asymptomatic, the shorter time one would be infectious.

The lethality rate, $\mu$, is clearly highly correlated to social, cultural, and economic factors other than the human bodily response. Although it was briefly discussed before, it is important to say that in perfect conditions for health care access, we would be able to draw a fixed number that depicts the average lethality rate. Nevertheless, Section 5 offers more information on both of the data sets we worked with here, even a decreasing lethality rate was observed for an increasing number of Coronavirus cases. Inevitably, we can infer some reasons for the increase such as different age groups more affected, or more hospital capacity available due to extra investment during the pandemic. But this type of effect is not very predictable and it would require superior data quality that would include information such as health care capacity and age group description etc. As a result, the model would become more complex, and so too would the analysis and the computational effort. For now, we are more interested in drawing the simplest model that properly fits the data and gives us extra insight in order to judge how the measures taken so far, if any, impact the disease dynamics. For the sake of simplicity, we will play a sad game and disregard the fact that one's living possibility depends not only on one's personal response to the infection but also the national response to health care of one's country.

To finish this discussion on the parameters and their meanings, one must note that although we keep a place for a loss of immunity with the parameter $\delta$, this parameter, if it is not zero, should not have an impact because it is expected that if any loss of immunity occurs, it should happen after at least 40 weeks, just like other coronaviruses. [1,5].

It is possible to study a scenario that might never happen, so due to this possibility, and eventual application, we present its impact in long run scenarios. 


\section{METHODOLOGY}

Once the modeling was done, we were left with three tasks: obtaining a numerical solution for approximating the solution of nonlinear ODE System, 2.1, determining values for the parameters and fitting the model to the available data, [4], in order to be able to make predictions.

The first task was quickly completed by implementing a 4th Order Runge-Kutta. Due to the system's nonlinearity, lower-order methods would not be sufficiently robust and the fact that Runge-Kutta methods are explicit make them computationally quite efficient.

The two last tasks are clearly entangled. As we discussed in the previous section, the parameters can be different for each population data set.

In order to fit the model, we had two major data sets: the number of new people that tested positive for the virus on a given day and the number of newly dead people on a given day. Both of these might be more or less unrealistic depending on the sub notification. For that reason, fitting the model to the data might be an ungrateful task, one might be trying to fit curves to mistaken numbers and therefore reaching poor conclusions. As we mentioned, to economize on tests, and because many of these people might not even be aware that they are infected, a great number of asymptomatic people is not accounted for. Therefore asymptomatic people have an important impact: they reduce the time of active pandemic because carrying the virus undetected speeds up the spread.

Then again, once the quality of the numerical approximation for the ODE system was assured, we did a few initial experiments with reasonable parameter values [3] in order to let the model's characteristics emerge. With the model, some indication of a few parameters, mostly the highly biologically correlated, and the data, we were able to infer which of the parameters left were crucial to capture the more unusual aspects of the data. And, for estimating those parameters, we recursively minimized nonlinear Least Square Problems, as we describe in the following Subsection.

\subsection{Parameters Estimation}

There is a large amount of data pointing to the most likely values for most of the parameters we need to estimate. But, due to the system's nonlinearity, subtle variations in the parameter values can drastically change the results. Therefore, from an initial estimation based on the literature, we decided to estimate parameter values by solving nonlinear least square problem(s). The available data for this purpose is the number of daily registered cases of deaths and infections, although there is no distinction between asymptomatic and symptomatic included in these numbers. We are going to assume that the registered cases of infections are relative to the symptomatic people because they are more likely to be tested. Now, if we call the daily registered number of infections, the daily cumulative number of infections, the daily registered number of deaths, and the cumulative number of deaths, $y_{i}, Y_{i}, z_{i}$ and $Z_{i}$, respectively, with $i$ being the $i^{\text {th }}$ day of infection and corresponding to the time interval $\left[t_{i-1}, t_{i}\right] ; y, Y, z$ and $Z$ are vectors whose entries carry the 
whole data available. By relaying in the numerical approximation for the solution of the ODE system which we obtained from our initial guess parameters, $\wp^{0}$, we get the vectors $S, L, A, I$, $R$ and $D$. Hence we expect $\delta I_{i}=\int_{t_{i-1}}^{t_{i}} I(t) \approx y_{i}, \sum^{i} \delta I_{i}=\int_{t_{0}}^{t_{i}} I(t) \approx Y_{i}, \delta D_{i}=\int_{t_{i-1}}^{t_{i}} D(t) \approx z_{i}$, and $\sum^{i} \delta D_{i}=\int_{t_{0}}^{t_{i}} D(t) \approx Z_{i}$. The numerical approximation for the solution will depend on the values of the parameters, therefore we aim to obtain the best set of parameters, $\wp *$, that will minimize $\|\delta I-y\|^{2},\left\|\sum \delta I-Y\right\|^{2}$ and $\left\|\sum \delta D-Z\right\|^{2}$, either separately or combined. For the minimization involving cumulative data, being symptomatic or deceased, $W_{Y_{i}}=1 / Y_{i}^{2}$ and $W_{Z_{i}}=1 / Z_{i}^{2}$ were taken as weights, respectively. Many experiments were carried out and the error measure worked as a guide to propose some initial guesses for parameters whose estimations were not available, such as $\alpha$ values or the lethality rate parameters.

\subsection{The Lethality Rate}

The daily number of deceased in both sets of data behaves in a rather unexpected way. There are clear oscillations decaying in amplitude in both data sets. Bearing this behaviour in mind we made the option for fitting the data taking the lethality as a damped oscillating function of time $\mu(t)=c_{1}+c_{2} \sin \left(\frac{t \pi}{c_{3}}+c_{4}\right) \cdot e^{\frac{-t \pi}{c_{5}}}$. In this case, we were left with the five constants $c_{1}, c_{2}$, $c_{3}, c_{4}, c_{5}$ to be found in the manner we described in previous subsection. While a high lethality rate may abruptly end a disease dynamic, as the virus destroys itself too fast while killing the host, a sufficiently mild lethality is more a product of the symptomatic cases than anything else. Regarding COVID-19, on the one hand, it is not so disruptive that it kills us at a very high rate. However, on the other hand, it has a sufficiently low lethality so that it keeps spreading and therefore can reach and be lethal to a greater number of people. For this reason, our proposed lethality function is not so much to make better predictions as it is to point to the fact that aspects usually unconsidered when modelling a disease, such as social ones, are playing an important role in the death toll. In addition, this kind of lethality rate is better to tune the fit to the other compartments. Nevertheless, once we extrapolate the data period for $t>>t_{0}$, in order to try to figure out some possible outcomes, this lethality function will behave as a constant. One can also think that if the disease has sufficiently spread, we will end up getting an average number for lethality despite social distinctions.

We are now ready to present the results.

\section{RESULTS}

Here we present some particularities from the data, optimised set of parameters, and respective approximations for the ODE System's solution for Brazil (Subsection 5.1); and Portugal (Subsection 5.2). In all the following experiments $\delta=0$ and the unit of time used is one day, $d$.

\subsection{Brazil}

We will start by presenting Brazil's set of parameters and respective fits. The first case was recorded for Brazil on February 26, 2020. The last date we use here is July 13, 2020. And 
$P_{\text {total }}=211785000$ people. The set of parameters, rounded to the hundredth, except for $\mu(t)$, related to the presented ODE System model 2.1 for Brazil is given by: $\beta=1 / 5 d^{-1} ; \gamma_{A}=0.09$ $d^{-1} ; \gamma_{I}=0.13 d^{-1} ; \sigma_{I}=0.87 ; v=2.11 ; \mu(t)=0.0015+0.0055 \sin \left(\frac{t \pi}{81}\right) e^{\frac{-t \pi}{316}} d^{-1} ;$ and $\alpha(t)$, in $(\text { person } * d)^{-1}$ units, given by

$$
\alpha(t)= \begin{cases}\alpha_{1}=0.59, & t \in[0,30[ \\ \alpha_{2}=0.09, & t \in[30,60[ \\ \alpha_{3}=0.12, & t \in[60,75[ \\ \alpha_{4}=0.07, & t \in[75,105[ \\ \alpha_{5}=0.06, & t \in[105,139]\end{cases}
$$

With these values, we obtained $\|\delta I-y\|^{2} \approx 8.7 e-008,\left\|\sum \delta I-Y\right\|^{2} \approx 19.01$ and $\left\|\sum D-Z\right\|^{2} \approx$ 5.91. Figures 1 to 4 show the results.
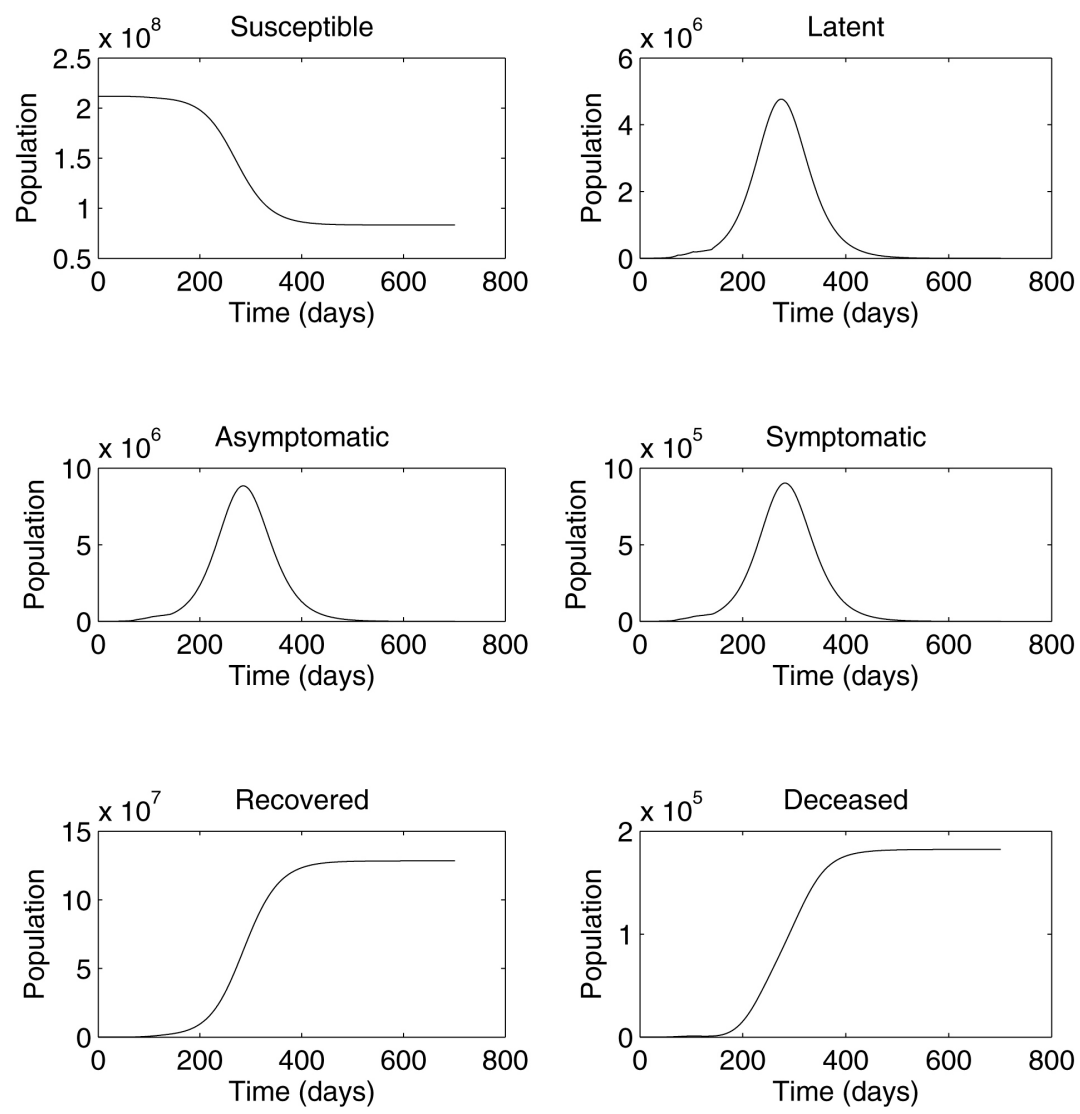

Figure 1: Numerical approximation for the ODE system solution with optimised set of parameters for Brazil. 


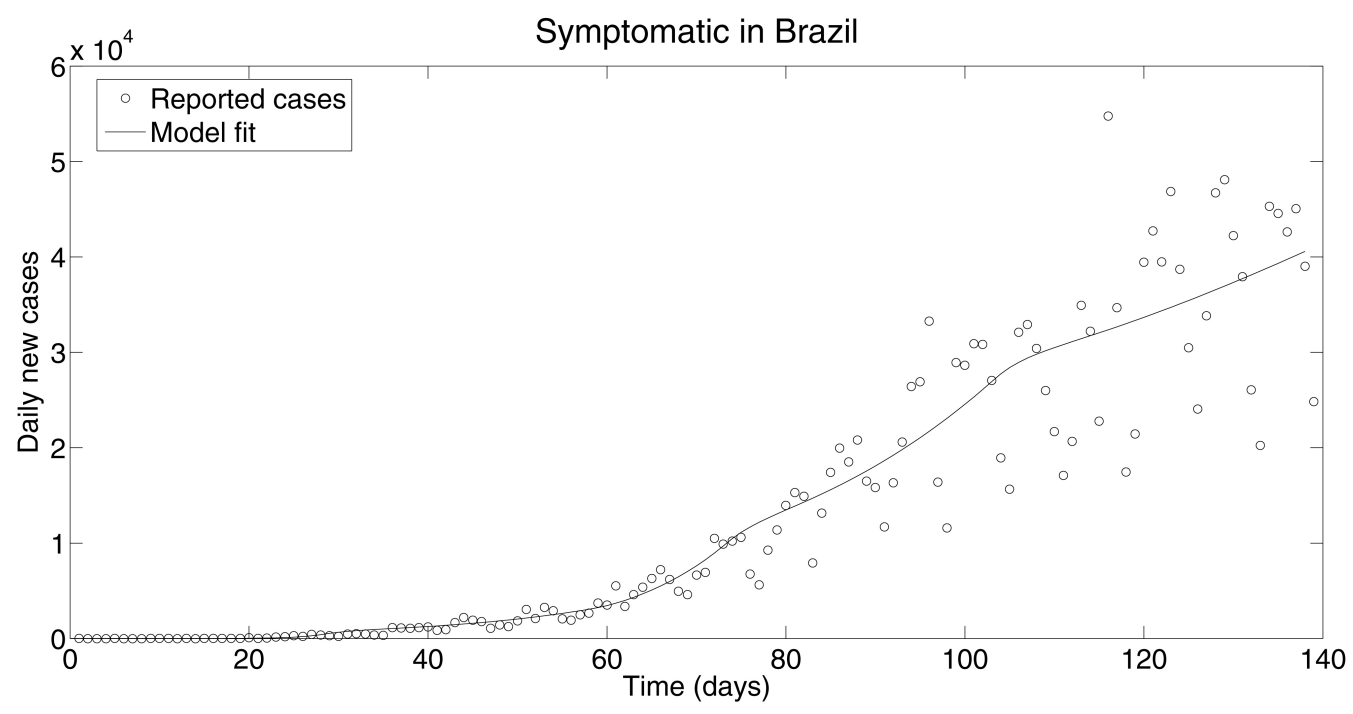

Figure 2: $y$ and $\delta I$ for Brazil.

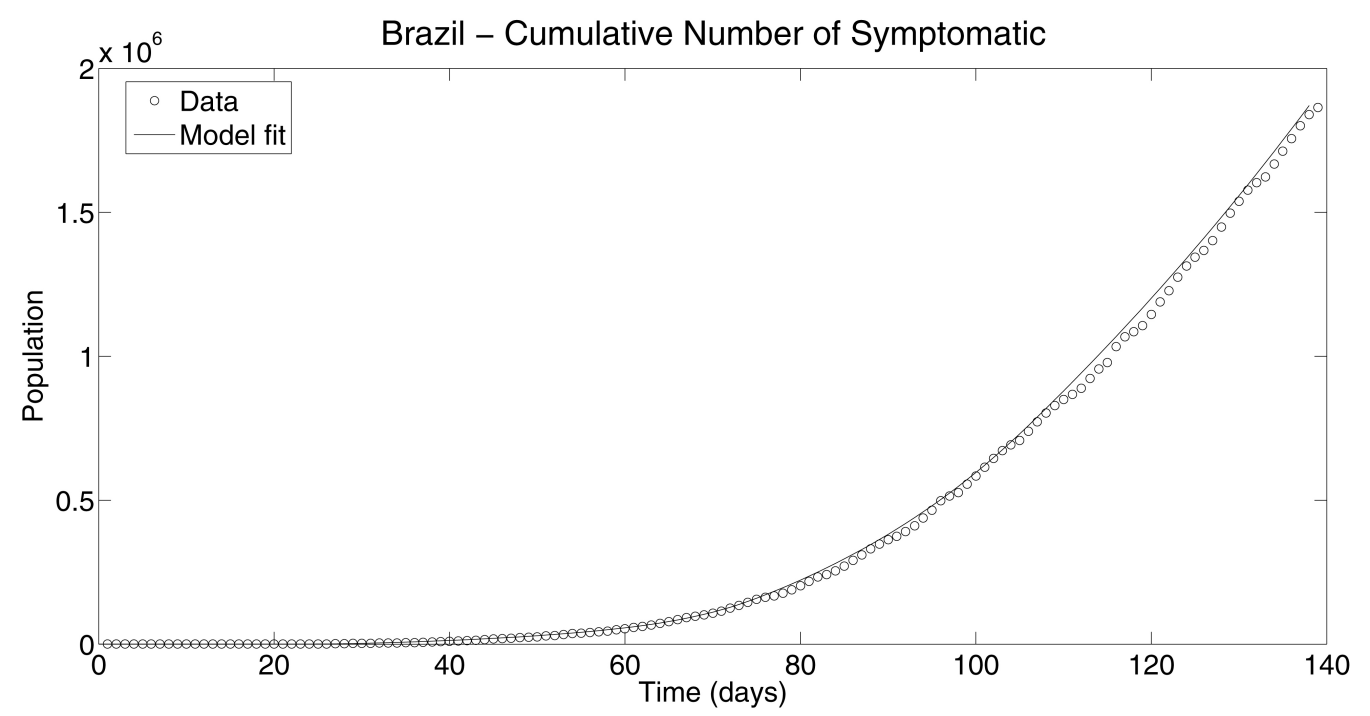

Figure 3: $Y$ and $\sum \delta I$ for Brazil. 


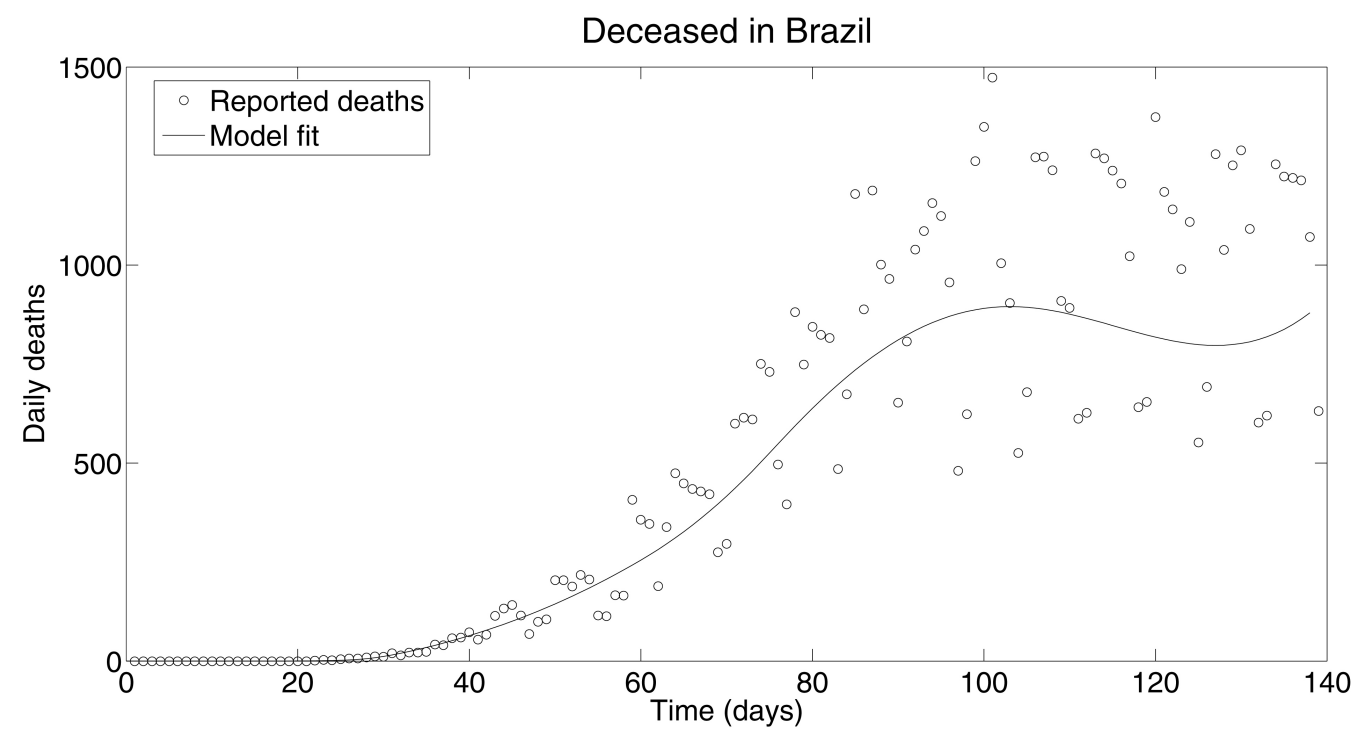

Figure 4: $z$ and $\delta D$ for Brazil.

\subsection{Portugal}

The first case recorded for Portugal took place on March 3rd, 2020. The last date we use here is July 13, 2020. And $P_{\text {total }}=10121000$ people. To fit Portugal's data, we started with the plausible parameters we used for, or obtained from, the Brazil experiments. In addition, we found plausible values for $\alpha(t)$ and the constants of $\mu(t)$ as explained in Subsections 4.1 and 4.2. Therefore, the results shown here were obtained with: $\beta=1 / 5 d^{-1} ; \gamma_{A}=1 / 10 d^{-1} ; \gamma_{I}=1 / 10 d^{-1} ; \sigma_{I}=0.87$; $v=2.11 ; \mu(t)=0.0003+0.0128 \sin \left(\frac{t \pi}{86.4818}+1.7754\right) e^{\frac{-t \pi}{129.7461}} d^{-1} ;$ and $\alpha(t)$, in $(\text { person } * d)^{-1}$ units, given by

$$
\alpha(t)= \begin{cases}\alpha_{1}=0.99, & t \in[0,20[ \\ \alpha_{2}=0.08, & t \in[20,30[ \\ \alpha_{3}=0.03, & t \in[30,40[ \\ \alpha_{4}=0.02, & t \in[40,65[ \\ \alpha_{5}=0.06, & t \in[65,100[ \\ \alpha_{6}=0.05, & t \in[65,133]\end{cases}
$$

With these values, we obtained $\|\delta I-y\|^{2} \approx 2.5 e-008,\left\|\sum \delta I-Y\right\|^{2} \approx 9.76$ and $\left\|\sum D-Z\right\|^{2} \approx$ 5.65. Figures 5 to 8 show the results.

Taking $\alpha(t)$ as a piecewise constant function was crucial in order to fit data. We understood that this was the most socially impacted and flexible parameter in the system. Therefore, it is necessary to show different possible disclosures for the pandemic by taking a $\alpha_{f}(t)$ as all or 

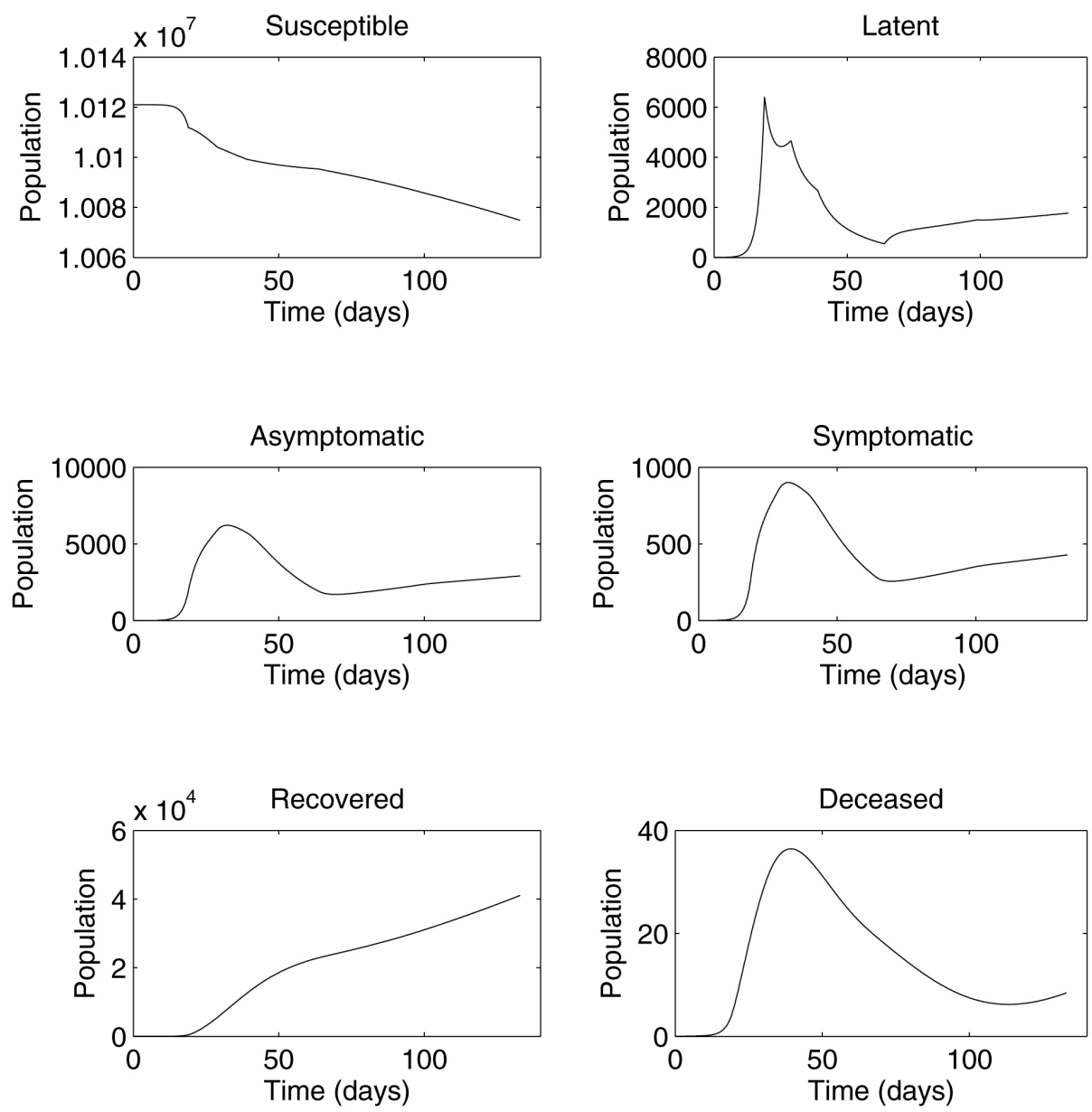

Figure 5: Numerical approximation for the ODE system solution with optimised set of parameters for Portugal.

some of the values the functions possibly assumed in previous intervals of time, for $t \geq 139$ days in Brazil see Figures 9 to 12, and for $t \geq 133$ days in Portugal, see Figures 13 to 16. 


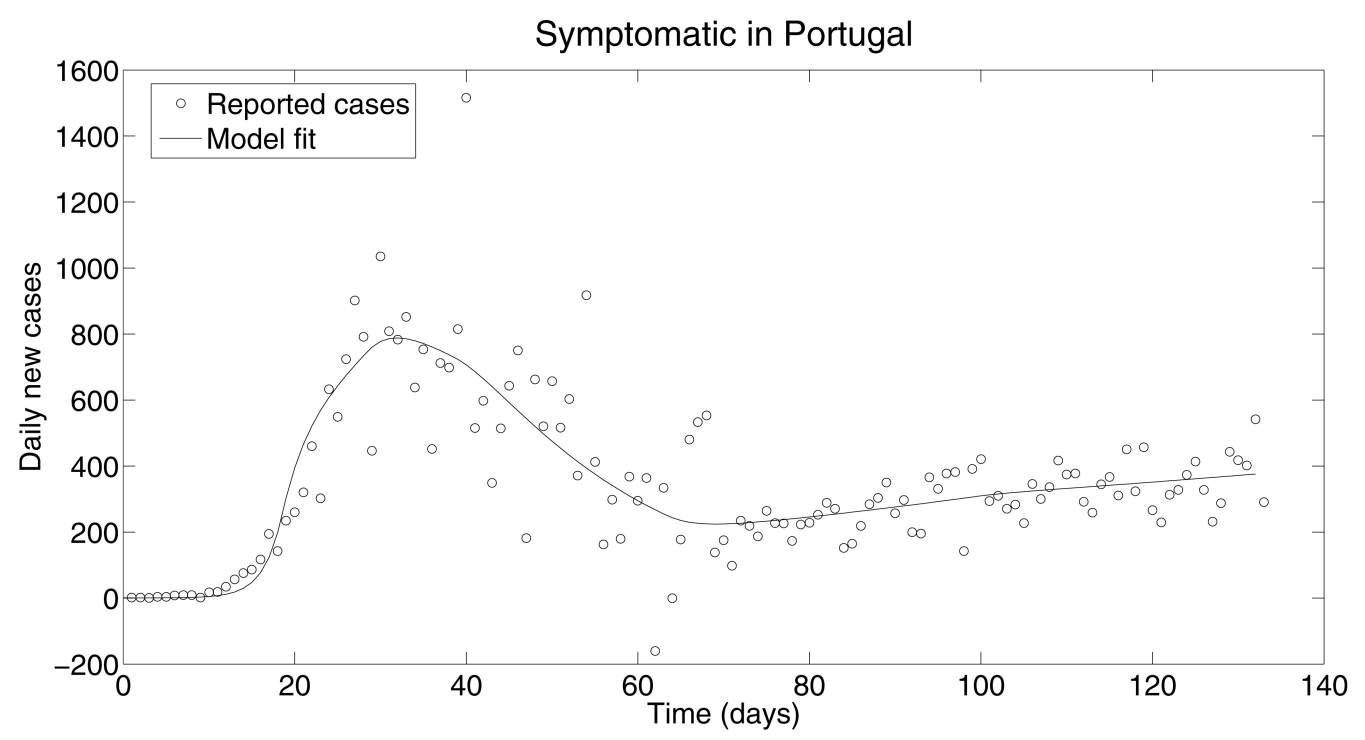

Figure 6: $y$ and $\delta I$ for Portugal.

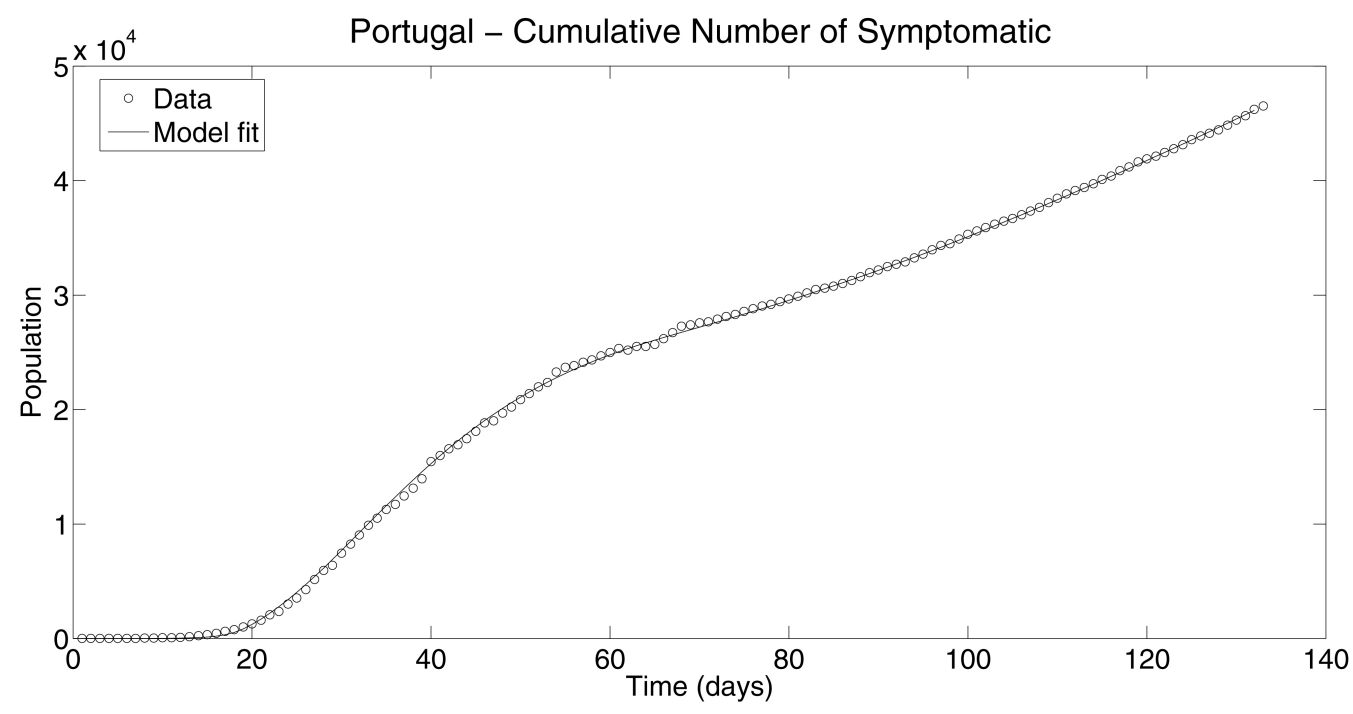

Figure 7: $Y$ and $\sum \delta I$ for Portugal. 


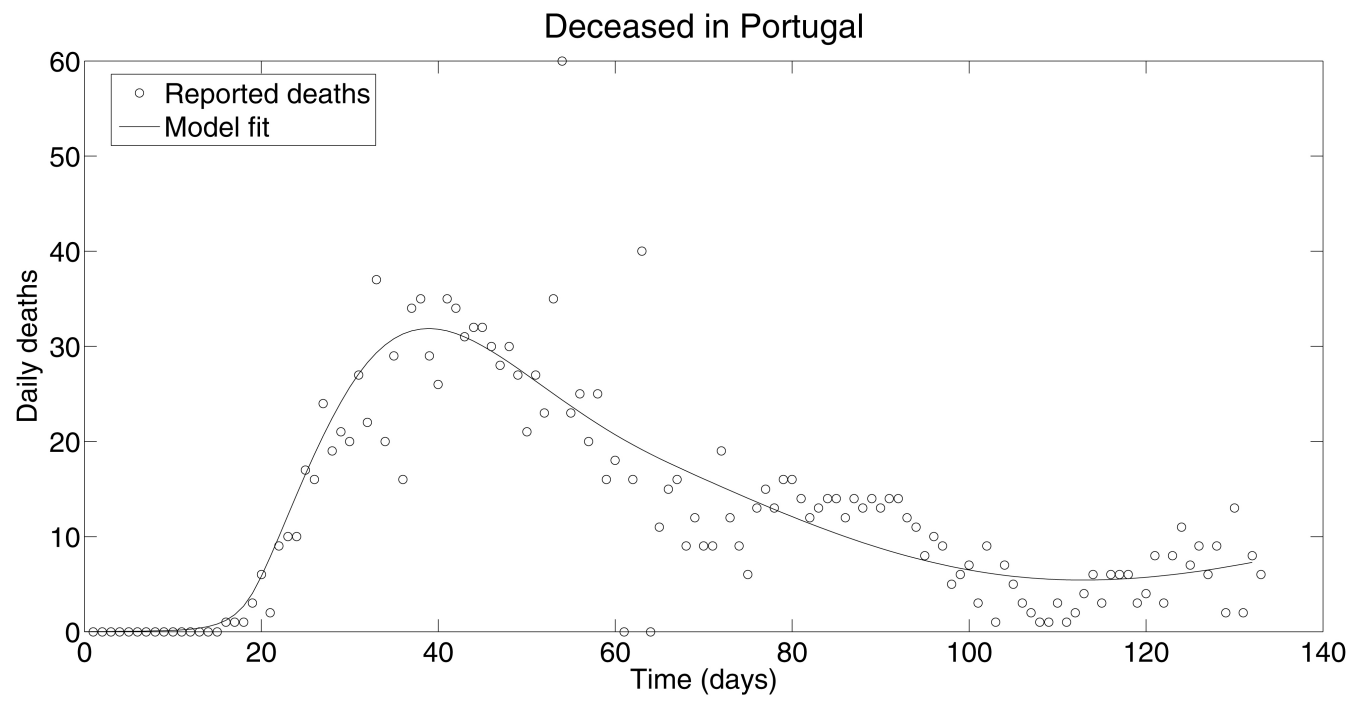

Figure 8: $z$ and $\delta D$ for Portugal.

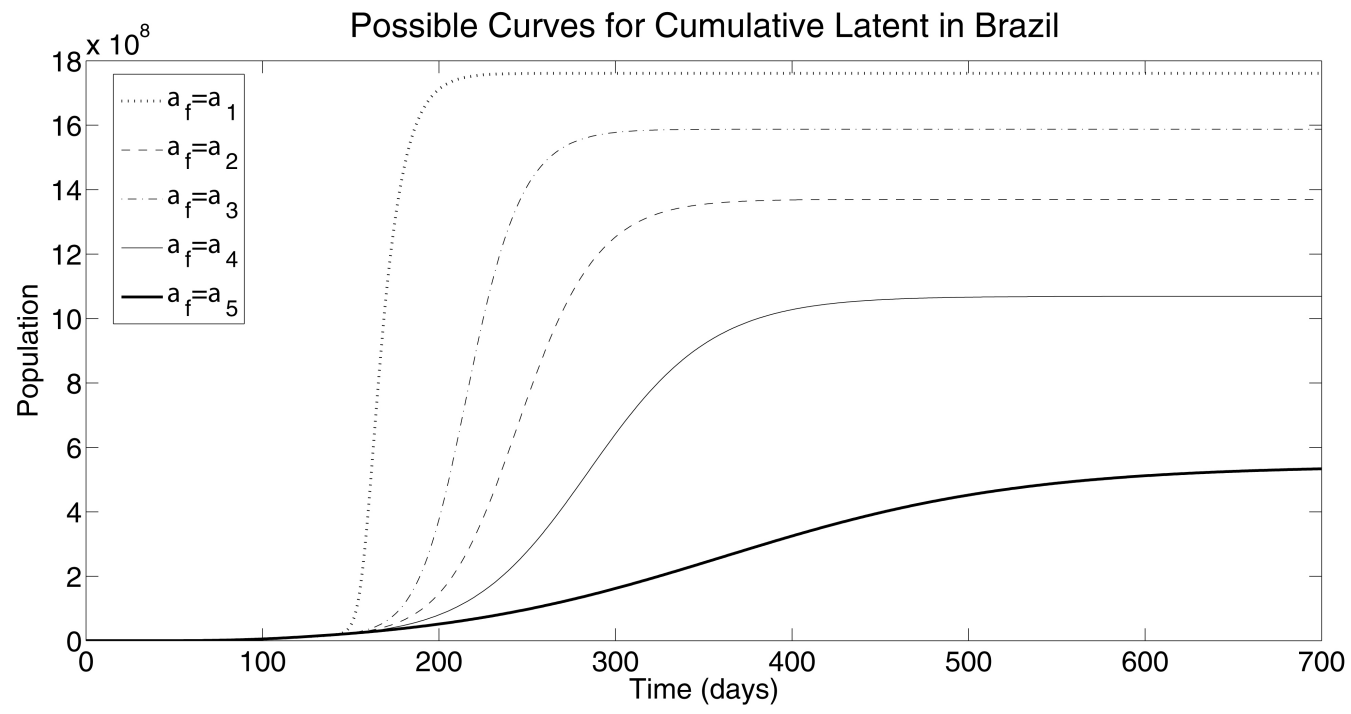

Figure 9: Predictions for the cumulative number of Infected people, despite the classification as symptomatic or asymptomatic, for different values of $\alpha_{f} ; \alpha_{1}=0.59, \alpha_{2}=0.09, \alpha_{3}=0.12$, $\alpha_{4}=0.07, \alpha_{5}=0.06$. 


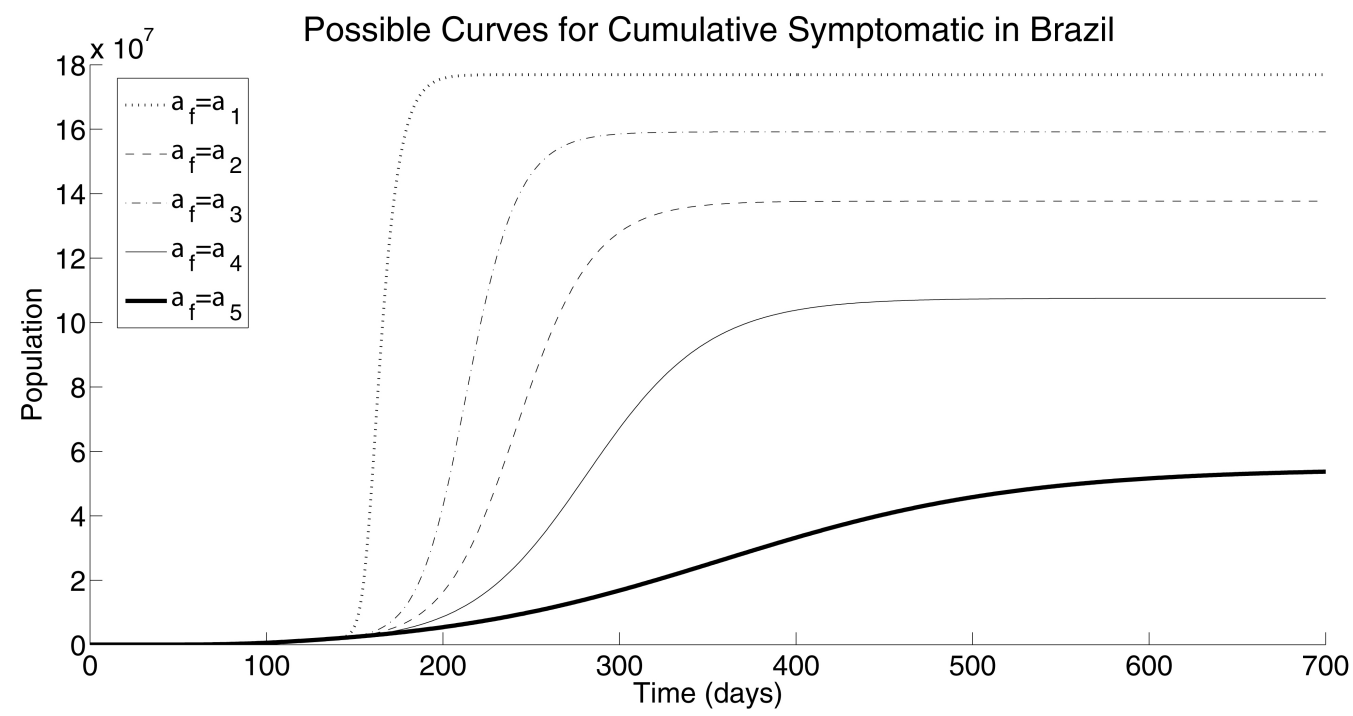

Figure 10: Predictions for the final number of Symptomatic people for different values of $\alpha_{f}$; $\alpha_{1}=0.59, \alpha_{2}=0.09, \alpha_{3}=0.12, \alpha_{4}=0.07, \alpha_{5}=0.06$.

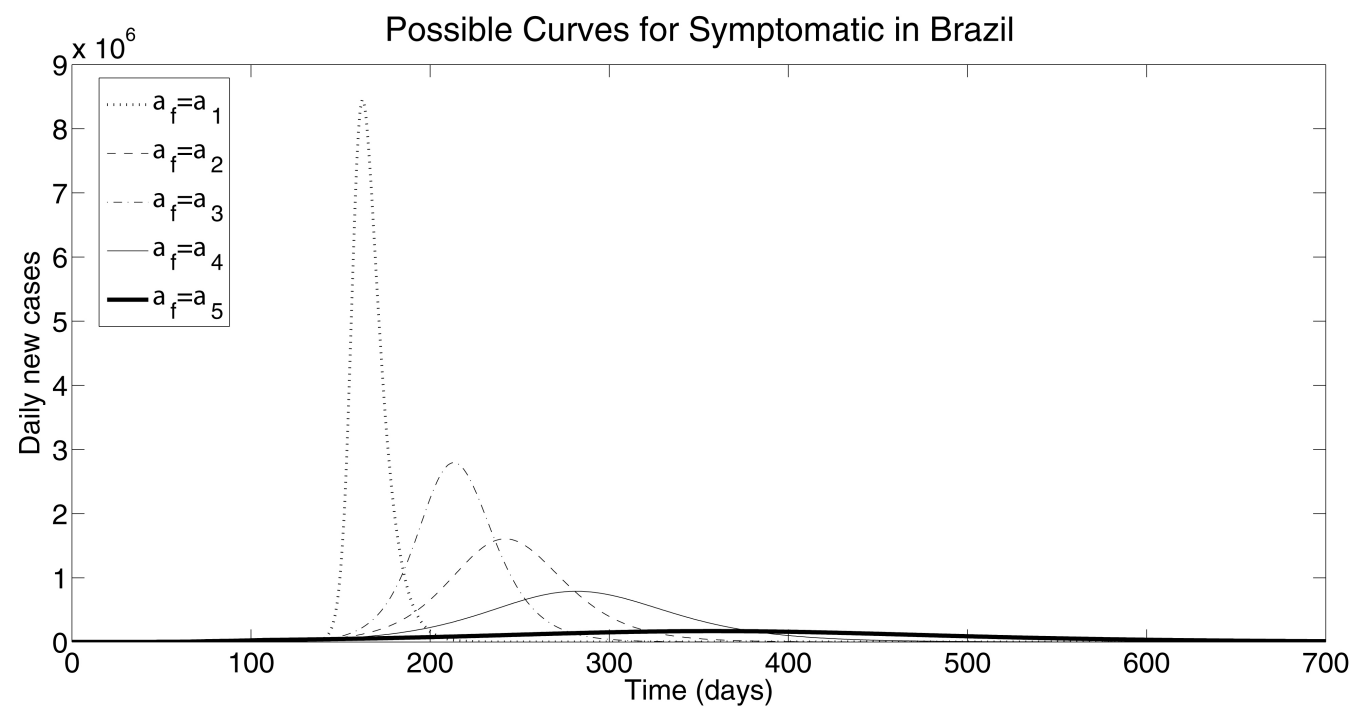

Figure 11: Predictions for the peak of Symptomatic people for different values of $\alpha_{f} ; \alpha_{1}=0.59$, $\alpha_{2}=0.09, \alpha_{3}=0.12, \alpha_{4}=0.07, \alpha_{5}=0.06$. 


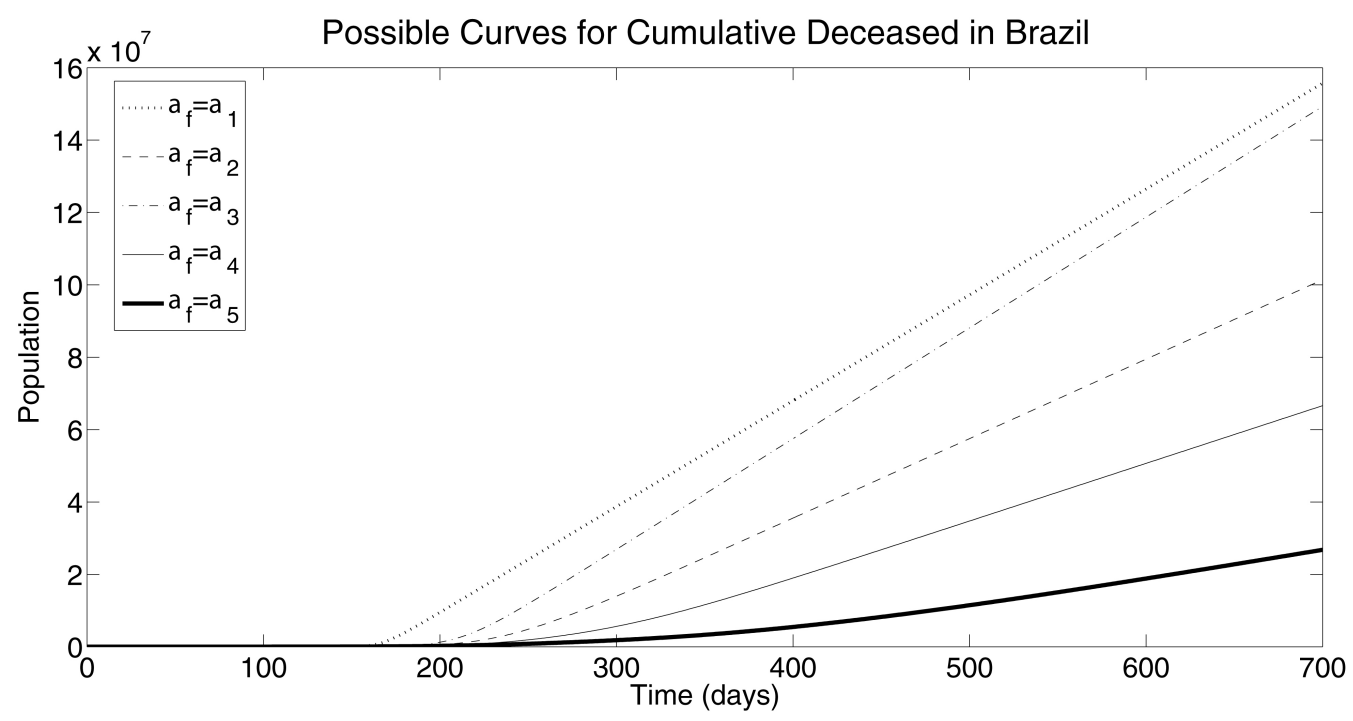

Figure 12: Predictions for the cumulative number of Deceased people for different values of $\alpha_{f}$; $\alpha_{1}=0.59, \alpha_{2}=0.09, \alpha_{3}=0.12, \alpha_{4}=0.07, \alpha_{5}=0.06$.

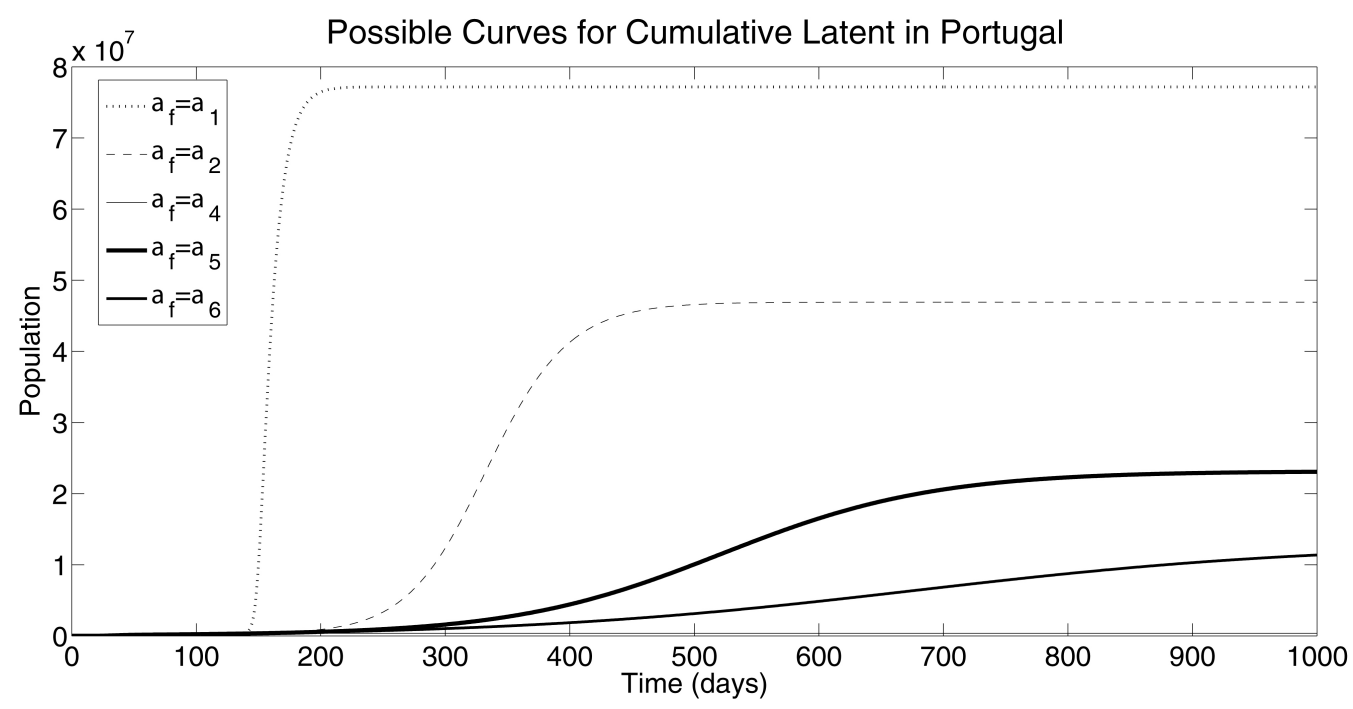

Figure 13: Predictions for the cumulative number of Infected people, despite the classification as symptomatic or asymptomatic, for different values of $\alpha_{f} ; \alpha_{1}=0.99, \alpha_{2}=0.08, \alpha_{3}=0.03$, $\alpha_{4}=0.02, \alpha_{5}=0.06, \alpha_{6}=0.05$. 


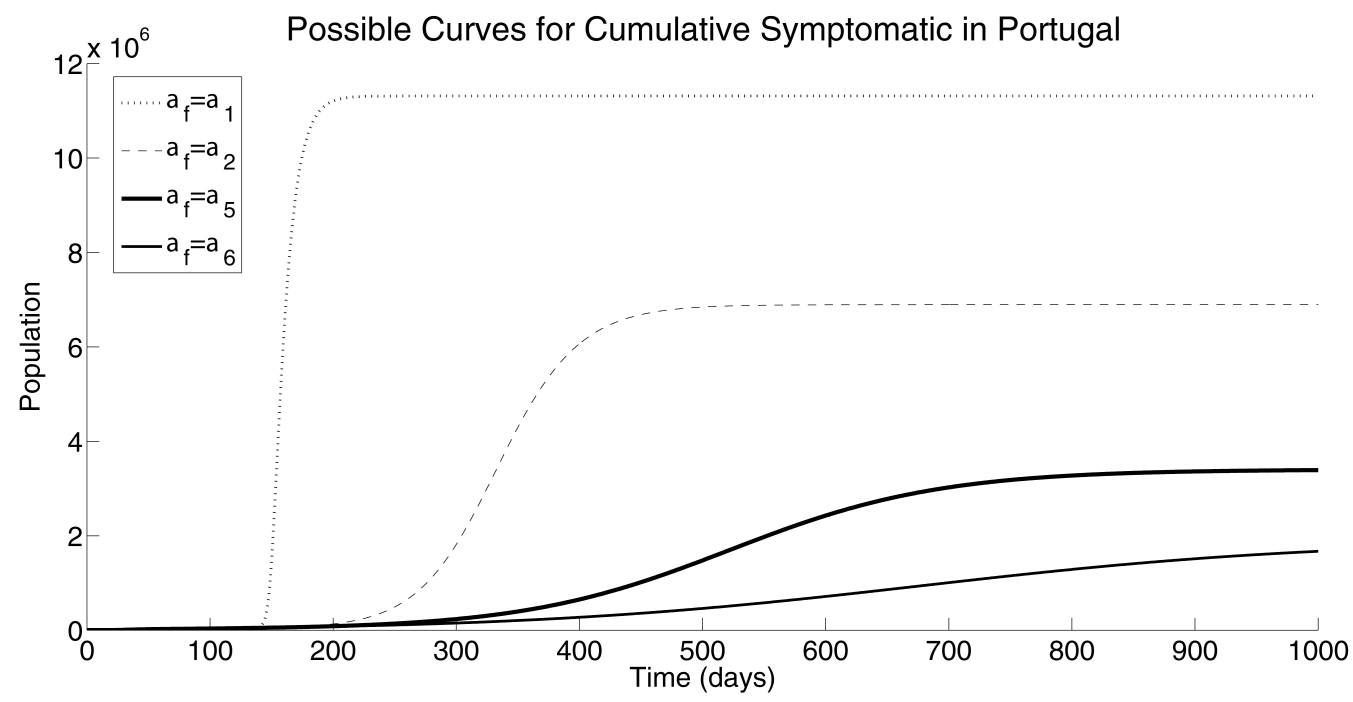

Figure 14: Predictions for the final number of Symptomatic people for different values of $\alpha_{f}$; $\alpha_{1}=0.99, \alpha_{2}=0.08, \alpha_{5}=0.06, \alpha_{6}=0.05$.

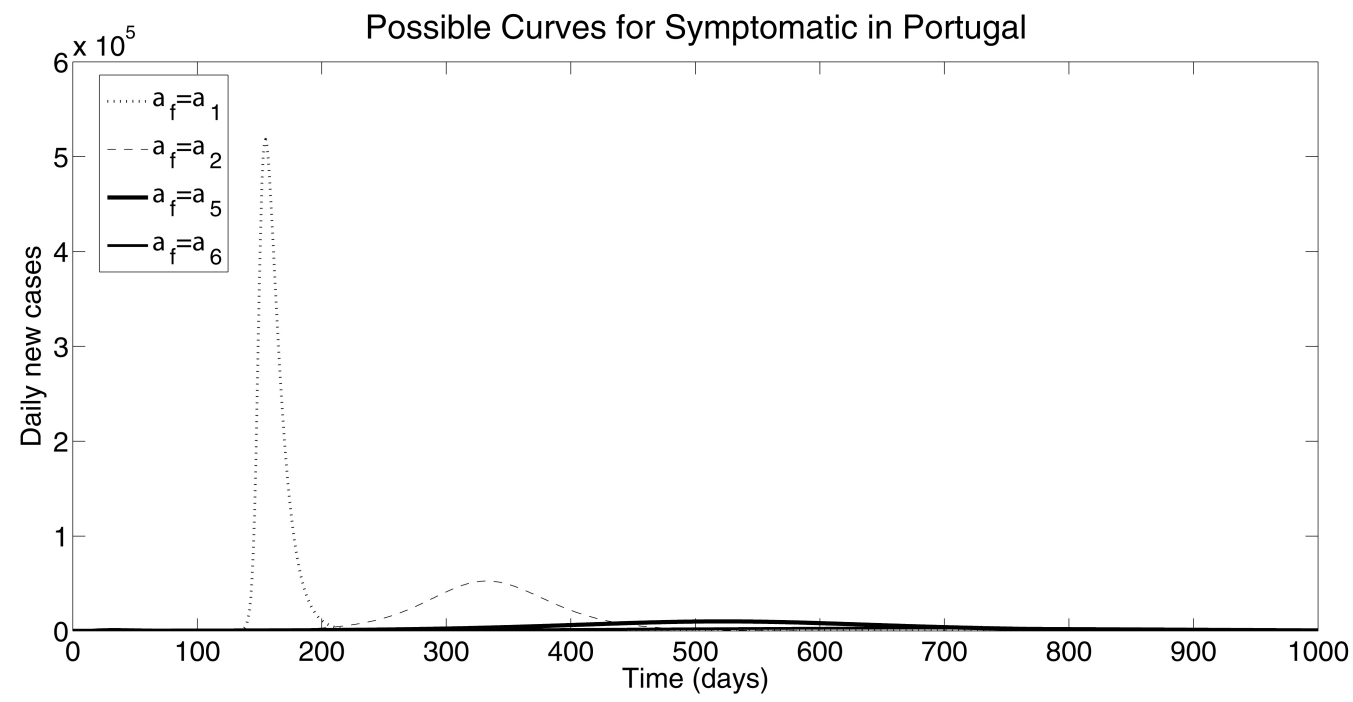

Figure 15: Predictions for the peak of Symptomatic people for different values of $\alpha_{f} ; \alpha_{1}=0.99$, $\alpha_{2}=0.08, \alpha_{5}=0.06, \alpha_{6}=0.05$. 


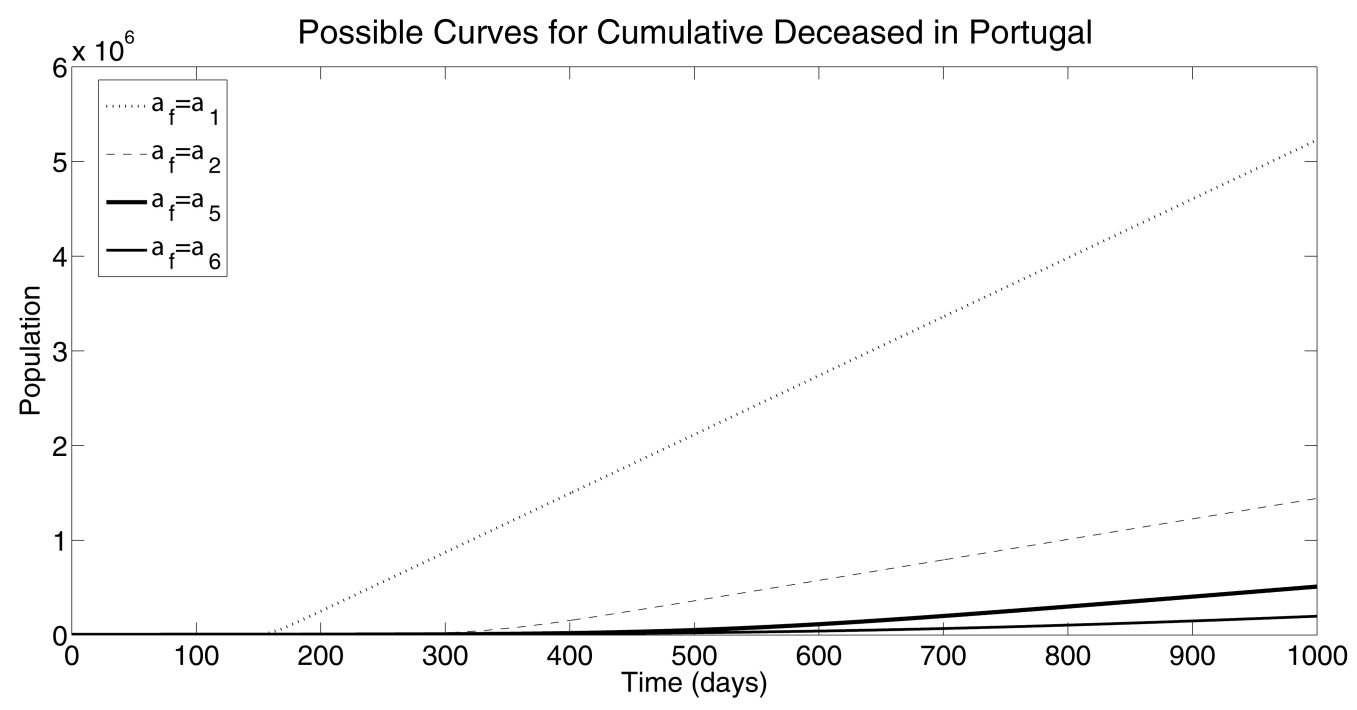

Figure 16: Predictions for the cumulative number of Deceased people for different values of $\alpha_{f}$; $\alpha_{1}=0.99, \alpha_{2}=0.08, \alpha_{5}=0.06, \alpha_{6}=0.05$.

\section{DISCUSSION}

As previously shown, we were able to estimate $v$ and $\sigma_{I}$ according to theoretical previsions. According to the results, approximately $87 \%$ of cases are asymptomatic and, therefore, are not usually accounted for in data. Compare Figures 9 and 10, for the Brazilian case, and Figures 13 and 14 , for the Portuguese case; the final number of infections is ten times greater if we take into account asymptomatic cases. Furthermore, $87 \%$ of cases infect more than twice as many people as the symptomatic group and that is the reason it is crucial that all individuals practice social distancing properly in order to lower contagion.

Figures 8 to 12, concerning Brazil, and Figures 13 to 14, concerning Portugal further display the impact of the parameter responsible for the virus transmission. It seems to be the case that Portugal started with $\alpha_{1} \approx 0.99$ for the first 20 days of infection and it was able to keep it below 0.08 ever since. For Brazil, the beginning was slower, possibly due to the social class of the initially infected, but even if this is the value that $\alpha(t)$ will assume from day 139 on, the number of cases and deceased is near four times what it could be if its final value, $\alpha_{5}=0.06$, is maintained. However, getting back to its $\alpha_{1}$ would mean that the peak is just ahead and the wave will be gone by the end of the year if $\delta$ is actually 0 .

Portugal was able to reach $\alpha_{3}=0.03$ and $\alpha_{4}=0.02$ during the lockdown period and if it returned to these values, the curve would flatten so much so that the pandemic would be active for a long time, that the results are omitted in Figures 13 to 14. 


\section{CONCLUSION}

The purpose of this research is to better understand a virus that matches perfectly contemporary human society in the sense of being able to spread silently and maximize its lethality in the absence of social restrictions.

There is not much room for doubts in at least one sense; the spreading pandemic and the human reaction to it are political. In an unethical manner, the faster, the better, and flattening peaks would only keep us under the restrictions necessary to lower the infection spread. In an approach that is guided by the idea that lives matter, and, then, having to think of how to live with the restrictions necessary so living matters, after all, we are social creatures, some deep reflections and, probably, modifications in our society's relation to capital would be necessary.

Portugal is being able to handle the transmission in an optimised manner, assuring hospital care for those who need it. The measures that are being taken in this country should teach us something.

We are not forgetting all the cultural, economic, and social differences between these two countries. We are only pointing to the fact that keeping the pandemic sufficiently under control in order to maximize the number of survivors is possible.

With all the difficulties that have been in the news, Brazil was able to keep lowering the spread up until $\alpha_{5}=0.06$. But around July 13, 2020, unlock measures were already starting in some Brazilian States. And that without regard to the country's possibility of receiving those who would need hospital care.

Overall, although the sets of parameters used in both experiments are certainly not the final set it is clearly possible to intervene in the disease dynamics.

\section{Acknowledgments}

A much considered thank you to the COVID-19 - Unicamp study group.

\section{REFERENCES}

[1] N.L. Bert, A.T. Tan \& K.K. et al. SARS-CoV-2-specific T cell immunity in cases of COVID-19 and SARS, and uninfected controls. Technical report, https://doi.org/10.1038/s41586-020-2550-z (2020).

[2] M. da Saude do Brasil. Concluida a maior pesquisa brasileira sobre a COVID-19. Technical report, https://www.saude.gov.br/noticias/agencia-saude/47155-concluida-a-maior-pesquisa-brasileirasobre-a-covid-19 (2020).

[3] C. for Disease Controll \& Prevention. COVID-19 Pandemic Planning Scenarios. Technical report, https://www.cdc.gov/coronavirus/2019-ncov/hcp/planning-scenarios.html (2020).

[4] E.C. for Disease Prevention \& Control. Download today's data on the geographic distribution of COVID-19 cases worldwide. Technical report, https://www.ecdc.europa.eu/en/publicationsdata/download-todays-data-geographic-distribution-covid-19-cases-worldwide (2020). 
[5] S.M. Kissler, C. Tedijanto, E. Goldstein, Y.H. Grad \& M. Lipsitch. Projecting the transmission dynamics of SARS-CoV-2 through the postpandemic period. Technical report, https://science.sciencemag.org/content/368/6493/860.full (2020).

[6] J.D. Murray. "Mathematical Biology: I". Interdisciplinay Applied Mathematics. Springer, New York (2002).

[7] J. Spring \& P. Fonseca. Brazil likely has 12 times more coronavirus cases than official count, study finds. Technical report, https://www.reuters.com/article/us-health-coronavirus-brazil-cases/brazillikely-has-12-times-more-coronavirus-cases-than-official-count-study-finds-idUSKCN21V1X1 (2020).

(cc) BY 